\title{
A Gyro Signal Characteristics Analysis Method Based on Empirical Mode Decomposition
}

\author{
Qinghua Zeng, ${ }^{1,2}$ Shanshan Gu, ${ }^{1,2}$ Jianye Liu, ${ }^{1,2}$ Sheng Liu, ${ }^{3}$ and Weina Chen ${ }^{1,2}$ \\ ${ }^{1}$ Jiangsu Key Laboratory of Internet of Things and Control Technologies, Nanjing University of Aeronautics and Astronautics, \\ 29 Jiangjun Road, Nanjing 211106, China \\ ${ }^{2}$ Navigation Research Center, College of Automation Engineering, Nanjing University of Aeronautics and Astronautics, \\ 29 Jiangjun Road, Nanjing 211106, China \\ ${ }^{3}$ AVIC Luoyang Electro-Optical Equipment Research Institute, Luoyang 471009, China
}

Correspondence should be addressed to Qinghua Zeng; zengqh@nuaa.edu.cn

Received 9 March 2016; Revised 14 June 2016; Accepted 22 June 2016

Academic Editor: Andrea Cusano

Copyright (C) 2016 Qinghua Zeng et al. This is an open access article distributed under the Creative Commons Attribution License, which permits unrestricted use, distribution, and reproduction in any medium, provided the original work is properly cited.

It is difficult to analyze the nonstationary gyro signal in detail for the Allan variance (AV) analysis method. A novel approach in the time-frequency domain for gyro signal characteristics analysis is proposed based on the empirical mode decomposition and Allan variance (EMDAV). The output signal of gyro is decomposed by empirical mode decomposition (EMD) first, and then the decomposed signal is analyzed by AV algorithm. Consequently, the gyro noise characteristics are demonstrated in the timefrequency domain with a three-dimensional (3D) manner. Practical data of fiber optic gyro (FOG) and MEMS gyro are processed by the AV method and the EMDAV algorithm separately. The results indicate that the details of gyro signal characteristics in different frequency bands can be described with the help of EMDAV, and the analysis dimensions are extended compared with the common AV. The proposed EMDAV, as a complementary tool of the AV, which provides a theoretical reference for the gyro signal preprocessing, is a general approach for the analysis and evaluation of gyro performance.

\section{Introduction}

As a key device for angular velocity detection, gyro plays an important role in inertial navigation system. However, the errors caused by the influence of gyro working environment will be accumulated with working time, and the navigation accuracy will be deteriorated [1]. Consequently, it is important to analyze the characteristic of gyro signal, which is helpful to identify the performance of the gyros.

The traditional Allan variance (AV) algorithm, which can reflect the characteristics of signal, is widely used to evaluate the performance of gyro $[2,3]$. However, it can only achieve the signal characteristics analysis in the time domain. Actually, due to the complexity of practical gyro signal, it is difficult to obtain the signal species and distributions comprehensively in time domain. So it is essential to analyze the gyro signal in the time-frequency domain.
Wavelet transform and empirical mode decomposition (EMD) are commonly adopted to implement the signal timefrequency analysis. Nonetheless, the problems of frequency overlapping, threshold instability, and decomposition and reconstruction algorithm errors existing in the wavelet transform bring some limitations for the gyro signal processing [4]. While being an adaptive local time-frequency analysis method [5], EMD does not possess the above disadvantages. The signal can be decomposed by its characteristic to a finite number of empirical mode functions by EMD, which is commonly applied in signal denoising [6], trend extraction [7], and so on.

In this paper, a gyro signal characteristic analysis approach based on the empirical mode decomposition and Allan variance (EMDAV) is proposed, which employs AV to analyze the decomposed gyro signal of EMD. Compared with the sole time domain characteristic of gyro signal obtained by 
traditional $\mathrm{AV}$, the proposed method enriches the real gyro performance evaluation approaches, supplies more detailed gyro signal characteristics information in the joint timefrequency domain, and provides the theoretical basis for the denoising threshold determination in signal preprocessing.

\section{Allan Variance Analysis}

As a common analysis tool in the time domain, AV is widely applied to identify the gyro errors characteristics. Assuming that there is a gyro signal sample sequence of $\{x(t)\}$ with the length of $N$ and the sampling frequency of $f$, its discrete-time AV can be expressed as [8]

$$
\sigma_{\mathrm{AVAR}}^{2}(\tau)=\frac{1}{2}\left\langle\left(\bar{x}_{t_{k}+\tau}(\tau)-\bar{x}_{t_{k}}(\tau)\right)^{2}\right\rangle,
$$

where the operator $\langle\cdot\rangle$ denotes ensemble averaging, $\bar{x}_{t_{k}}(\tau)$ is the averaged measure of FOG signal at time $t_{k}$, and $\tau=N / f$ is observation interval.

It is assumed that the various error sources are statistically independent; then the AV can be described as the sum of squares of quantization noise, angle random walk, bias instability, rate random walk, and rate ramp $[9,10]$ :

$$
\begin{aligned}
\sigma^{2}(\tau)= & \sigma_{\mathrm{QN}}^{2}(\tau)+\sigma_{\mathrm{ARW}}^{2}(\tau)+\sigma_{\mathrm{BI}}^{2}(\tau)+\sigma_{\mathrm{RRW}}^{2}(\tau) \\
& +\sigma_{\mathrm{RR}}^{2}(\tau) \\
= & \frac{3 Q^{2}}{\tau^{2}}+\frac{N^{2}}{\tau}+\frac{2 B^{2}}{\pi} \ln 2+\frac{K^{2} \tau}{3}+\frac{R^{2} \tau^{2}}{2},
\end{aligned}
$$

where $\sigma_{\mathrm{ARW}}, \sigma_{\mathrm{RRW}}, \sigma_{\mathrm{BI}}, \sigma_{\mathrm{QN}}$, and $\sigma_{\mathrm{RR}}$ are the variances of the above five kinds of errors separately and $Q, N, B, K$, and $R$ are the corresponding AV coefficients.

\section{EMDAV Analysis}

The traditional AV analysis method which presents the gyro noise characteristics in the only time domain cannot reflect its distributions among different frequency bands. However, the noise characteristics vary with the frequency. To reveal more details of the gyro noise characteristic, the EMDAV method is proposed in this paper.

As a method utilized to analyze the nonlinear and nonstationary signal, EMD decomposes the signal on the basis of time scale characteristics. There is no need to set the basic functions in advance for EMD which can decompose any type of signal. Thus EMD has obvious advantages in processing nonlinear and nonstationary signal.

The EMDAV algorithm proposed in the paper is based on the EMD and AV. After decomposing the gyro signal with EMD, the AV can be obtained with the help of intrinsic mode function (IMF). Lastly the AV logarithmic curve over time and frequency can be depicted. The detailed algorithm flow chart is shown in Figure 1.

The steps of the EMDAV based on gyro signal characteristics analysis method can be summarized as follows:

(1) Find all the local maxima and minima of the original signal $x(t)$.

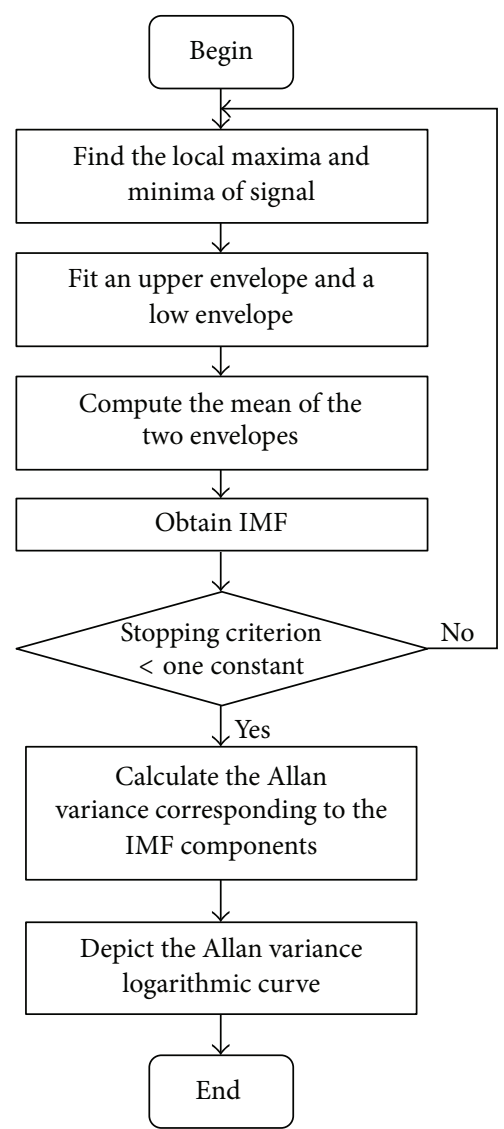

FIGURE 1: Flow chart of AV algorithm based on EMD.

(2) Fit the estimated points of the first step using natural cubic spline to form an upper envelope of $e_{\max }(t)$ and a lower envelope of $e_{\min }(t)$.

(3) Compute the mean of the two envelopes:

$$
m_{i}(t)=\frac{e_{\max }(t)+e_{\min }(t)}{2},
$$

where $i=1,2, \ldots, n ; n$ is the number of decomposition levels.

(4) Refine the estimate $h_{i}(t)$ of the IMF by subtracting the mean solved in the previous step from the current signal:

$$
h_{i}(t)=x_{i}(t)-m_{i}(t)
$$

where

$$
x_{i}(t)= \begin{cases}x(t), & i=1, \\ x(t)-\sum_{j=1}^{i-1} h_{j}(t), & i \geq 2 .\end{cases}
$$

(5) Proceed from step (1) again unless a stopping criterion has been fulfilled. The stopping criterion is [11]

$$
\mathrm{SD}=\sum_{t=0}^{T}\left[\frac{\left|h_{i-1}(t)-h_{i}(t)\right|^{2}}{h_{i-1}^{2}(t)}\right],
$$




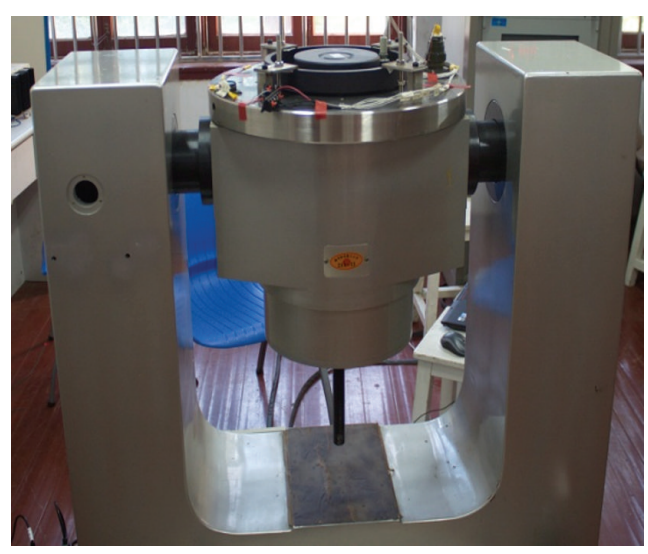

(a)

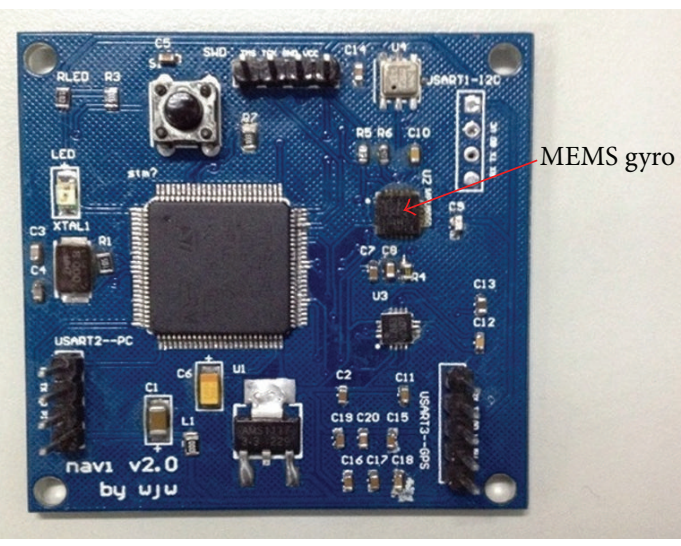

(b)

FIgURE 2: FOG and MEMS gyros: (a) FOG on turntable and (b) MEMS gyro.

where SD is a screening threshold. The iteration stops when $\mathrm{SD}$ is smaller than a constant. The constant value can be set between 0.2 and 0.3 [12].

Eventually, the IMF components of $h_{1}(t), h_{2}(t), \ldots, h_{n}(t)$ and the remainder of $r_{n}(t)$ can be obtained. Then the original signal can be expressed as

$$
x(t)=\sum_{i=1}^{n} h_{i}(t)+r_{n}(t) .
$$

(6) Calculate the AV coefficients corresponding to the IMF components and analyze the signal characteristics in different frequency bands.

From the above EMDAV procedures, we can see that the IMF of $h_{i}(t)$ indicates the different frequency components. With the increase of decomposition level, the frequency of decomposed signal decreases gradually. Namely, the first IMF represents the highest frequency component of the original signal. The frequencies of subsequent IMFs reduce almost in the form of the negative power of 2 [13] until the frequency of the remainder is very small. Consequently, each IMF corresponds to the signal component of different frequency.

Compared with the traditional AV analysis method, the characteristics and distributions of gyro signal in different frequency bands can be depicted in detail with EMDAV algorithm. One more piece of dimensional information can be shown in three-dimensional (3D) logarithmic graph of EMDAV compared to that in $2 \mathrm{D}$ curve of traditional AV.

\section{Experiment Results and Analysis}

To verify the algorithm proposed in this paper, AV and EMDAV are compared to analyze the FOG and MEMS gyro signals. Practical fiber optical gyro (FOG) and microelectromechanical system (MEMS) gyro static signals are acquired with the sampling frequency of $100 \mathrm{~Hz}$ and the sampling duration of $600 \mathrm{~s}$. The scene of experiment is shown in Figure 2.

As we all know, traditional AV coefficients come from original gyro signal, and we can compare them with EMDAV coefficients at the same time. Figure 3 shows the original signals and parts of IMF components decomposed by EMD. It can be seen that the characteristics of FOG and MEMS gyro signals are similar: the first level of decomposed signal belongs to high frequency part, and the frequency decreases with the increasing of decomposition level.

The original signals and IMF components decomposed by EMD are analyzed with AV, and corresponding coefficients (AV coefficients and EMDAV coefficients) are plotted in a logarithmic graph as shown in Figure 4. From Figure 4 we can find that the 16 curves in the first group from top to bottom are the AV logarithm curves of MEMS gyro original signal (the 0 level, traditional AV) and IMFs (1-15 levels, EMDAV), respectively. Because of the poor accuracy of MEMS gyro, the biggest $\mathrm{AV}$ value reaches $10^{5}$. The 16 profiles in the second group are the respective AV logarithm curves of FOG gyro original signal (the 0 level, traditional AV) and IMFs (EMDAV), where the biggest AV value reaches 1 . Taking the $\mathrm{AV}$ values at correlation time of $0.01 \mathrm{~s}$ for comparison, the maximum is from the original signal for both gyros, by which the first level of decomposed signals followed. The AV values decrease until the decomposition level increases to the last one, whose value corresponds to the minimum.

In addition, it can be seen from Table 1 and Figure 4 that the signal distributions of FOG and MEMS gyro are similar; that is to say, with the increase of decomposition level, the proportion of angle random walk is reduced while the proportion of rate random walk is increased. Also it is obvious that angle random walk is dominant in the high frequency signal while rate random walk accounts for a large proportion in the low frequency signal. In consequence, gyro signal can be denoised specifically with the demand of the navigation system.

Figure 5 shows the relationship between AV and EMDAV coefficients, where the zero level means the AV coefficient of original gyro signals. As mentioned before, the frequency of each IMF is decreased in the form of negative power of 2 gradually, which indicates the fact that the higher the EMD decomposition levels the smaller the signal frequency. It also 

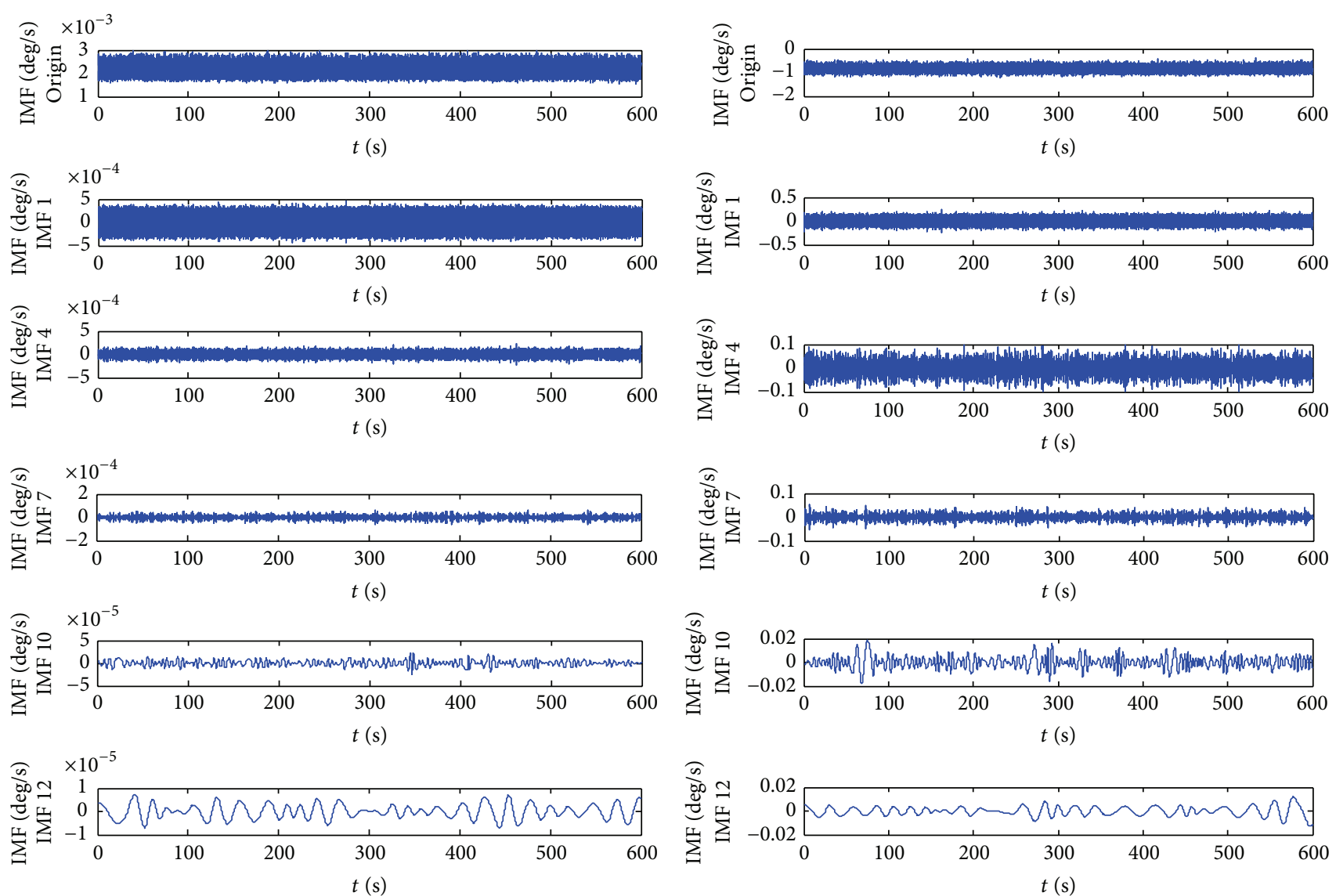

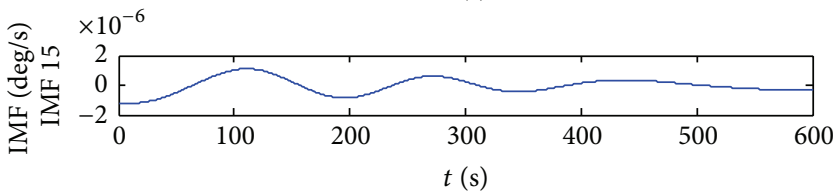

(a)

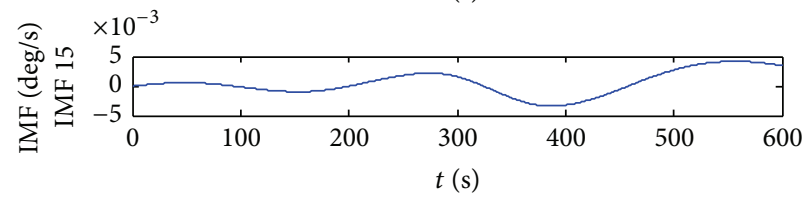

(b)

FIGURE 3: IMFs from EMD of gyro signals: (a) EMD of FOG signal and (b) EMD of MEMS gyro signal.

TABLE 1: AV coefficients of gyro signals on some frequencies.

\begin{tabular}{|c|c|c|c|c|c|c|c|}
\hline \multirow{2}{*}{\multicolumn{3}{|c|}{ Signal }} & \multicolumn{5}{|c|}{ AV coefficients } \\
\hline & & & $N\left({ }^{\circ} / \mathrm{h}^{1 / 2}\right)$ & $B(\% / \mathrm{h})$ & $K\left({ }^{\circ} / \mathrm{h}^{3 / 2}\right)$ & $R\left({ }^{\circ} / \mathrm{h}^{2}\right)$ & $Q(\mu \mathrm{rad})$ \\
\hline \multirow{6}{*}{ FOG } & \multicolumn{2}{|c|}{ AV (original signal) } & $1.40 e-3$ & $5.71 e-1$ & $1.22 e 1$ & $4.43 e 1$ & $3.55 e-2$ \\
\hline & \multirow{5}{*}{$\begin{array}{l}\text { EMDAV (signals } \\
\text { decomposed by } \\
\text { EMD) }\end{array}$} & Level 1 & $1.60 e-3$ & $4.64 e-1$ & $9.84 e 0$ & $3.56 e 1$ & $3.76 e-2$ \\
\hline & & Level 4 & $4.15 e-4$ & $1.75 e-1$ & $3.95 e 0$ & $1.44 e 1$ & $7.40 e-3$ \\
\hline & & Level 7 & $1.33 e-4$ & $6.66 e-2$ & $1.20 e 0$ & $4.25 e 0$ & $1.90 e-3$ \\
\hline & & Level 10 & $2.19 e-5$ & $1.02 e-2$ & $3.20 e-1$ & $1.24 e 0$ & $3.17 e-4$ \\
\hline & & Level 15 & $4.32 e-7$ & $2.10 e-4$ & $2.36 e-2$ & $7.72 e-2$ & $6.15 e-6$ \\
\hline \multirow{6}{*}{ MEMS gyro } & \multicolumn{2}{|c|}{ AV (original signal) } & $5.91 e-1$ & $3.10 e 1$ & $6.20 e 2$ & $2.24 e 3$ & $1.39 e 0$ \\
\hline & \multirow{5}{*}{$\begin{array}{l}\text { EMDAV (signals } \\
\text { decomposed by } \\
\text { EMD) }\end{array}$} & Level 1 & $1.37 e-1$ & $1.41 e 1$ & $2.10 e 2$ & $7.15 e 2$ & $8.78 e 0$ \\
\hline & & Level 4 & $1.01 e-1$ & $9.85 e 1$ & $2.16 e 3$ & $7.85 e 3$ & $2.37 e 0$ \\
\hline & & Level 7 & $9.93 e-2$ & $4.92 e 1$ & $8.50 e 2$ & $2.99 e 3$ & $1.40 e 0$ \\
\hline & & Level 10 & $1.59 e-2$ & $7.36 e 0$ & $2.54 e 2$ & $9.81 e 2$ & $2.30 e-1$ \\
\hline & & Level 15 & $1.30 e-3$ & $6.12 e-1$ & $6.54 e 1$ & $1.80 e 2$ & $1.81 e-2$ \\
\hline
\end{tabular}


TABLE 2: Performance contrasts between AV and EMDAV.

\begin{tabular}{lcc}
\hline & AV & EMDAV \\
\hline Signal & Stationary signal & Stationary and nonstationary signal \\
Result presentation (fingerprint) & $2 \mathrm{D}$ & 3D \\
Domain of signal analysis & Time domain & Time-frequency domain \\
Ability of signal identification at different frequencies & No & Yes \\
Numbers of obtained parameters can be referenced & Less & More \\
Reference for signal denoising & No & Yes \\
\hline
\end{tabular}

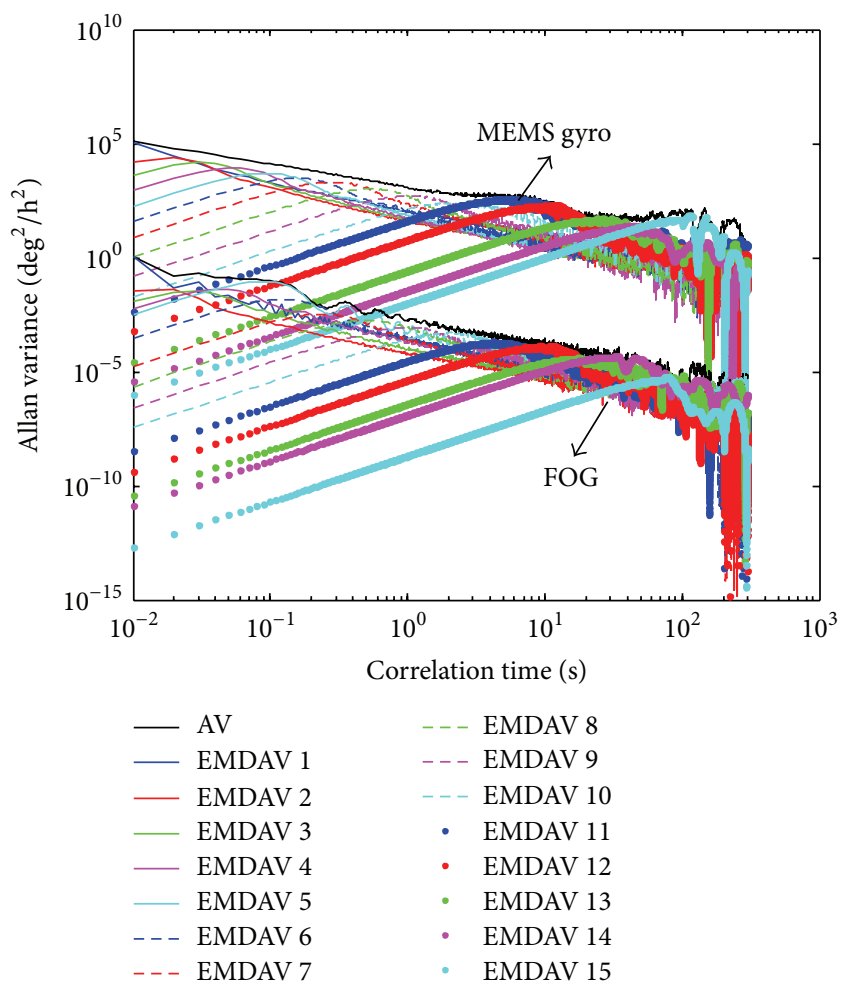

FIgURE 4: Logarithmic diagram of AV and EMDAV.

shows that the EMDAV coefficients trend to decrease with the decrease of signal frequency.

From the comparison of Figures 5(a) and 5(b), the coefficients of MEMS gyro are much larger than that of FOG; namely, the accuracy of MEMS gyro is worse than FOG. As for FOG (in Figure 5(a)), the EMDAV coefficients in the 5th level are larger, which means that the angle random walk noise, bias instability noise, rate random walk noise, and rate ramp noise are more dominant. The EMDAV coefficients of the 8th level and higher level are very small, and the EMD denoising method will be effective when the decomposition levels are larger than 7. As for MEMS gyro (in Figure 5(b)), the EMDAV coefficients are dominant in the 2nd level and are largest for 4 parameters, and reference decomposition level of 9 will be applicable for EMD denoising method. Therefore the algorithm proposed in this paper can provide guidance for gyro denoising.

The relationships among AV values of IMFs, correlation time, and EMD decomposition levels are shown in the AV and EMDAV 3D logarithmic graph of Figure 6, where the top curves are AV values of MEME gyro while the bottom are of FOG. Traditional AV logarithmic diagram is the 2D curve on the 0 level, and EMDAV logarithmic diagram is the $3 \mathrm{D}$ view on 1-15 levels. It can be found that though the $\mathrm{AV}$ values of MEMS gyro are larger than that of FOG, the signal magnitudes of the both gyros tend to reduce with the decrease of frequency. The 3D figures of both FOG and MEMS gyro signals show the special characteristics of the FOG and MEMS gyro performance. The AV values decrease with the increase of EMD levels and correlation time. The ridges, which also can be seen from Figure 4, are the collections of the points with a zero slope in logarithmic diagram of every IMF signal. Obviously the ridge of FOG signal is consistent with that of MEMS gyro signal.

The contrasts between AV and EMDAV are listed in Table 2. As can be seen from the table, EMDAV can analyze the both stationary and nonstationary signals with the analysis results showing the $3 \mathrm{D}$ relationship (served as the 3D fingerprint of a gyro) among AV, time, and frequency. In addition, various kinds of noises can be identified to provide more details about the signal characteristics of gyro. EMDAV can obtain more referable parameters than $\mathrm{AV}$ and provide a reference for signal denoising.

\section{Conclusion}

An AV analysis algorithm based on EMD for gyro error is proposed in this paper. The AV value is calculated by the signal decomposed based on the characteristic time scale, and the signal characteristics of gyro error in different frequency bands are revealed. Theoretical analysis and experiments of practical FOG and MEMS gyro data indicate the following:

(1) Compared with the characteristic of gyro error in the sole time domain given by the traditional AV, the EMDAV algorithm can describe the distributions on different kinds of gyro signals in time-frequency domain.

(2) The EMDAV algorithm extends the dimensions of gyro signal characteristics analysis and hence obtains more parameters than traditional AV.

(3) The EMDAV algorithm is able to determine the decomposition level for gyro signal denoising and provide a theoretical guidance to the gyro signal denoising and other preprocessing algorithms. 

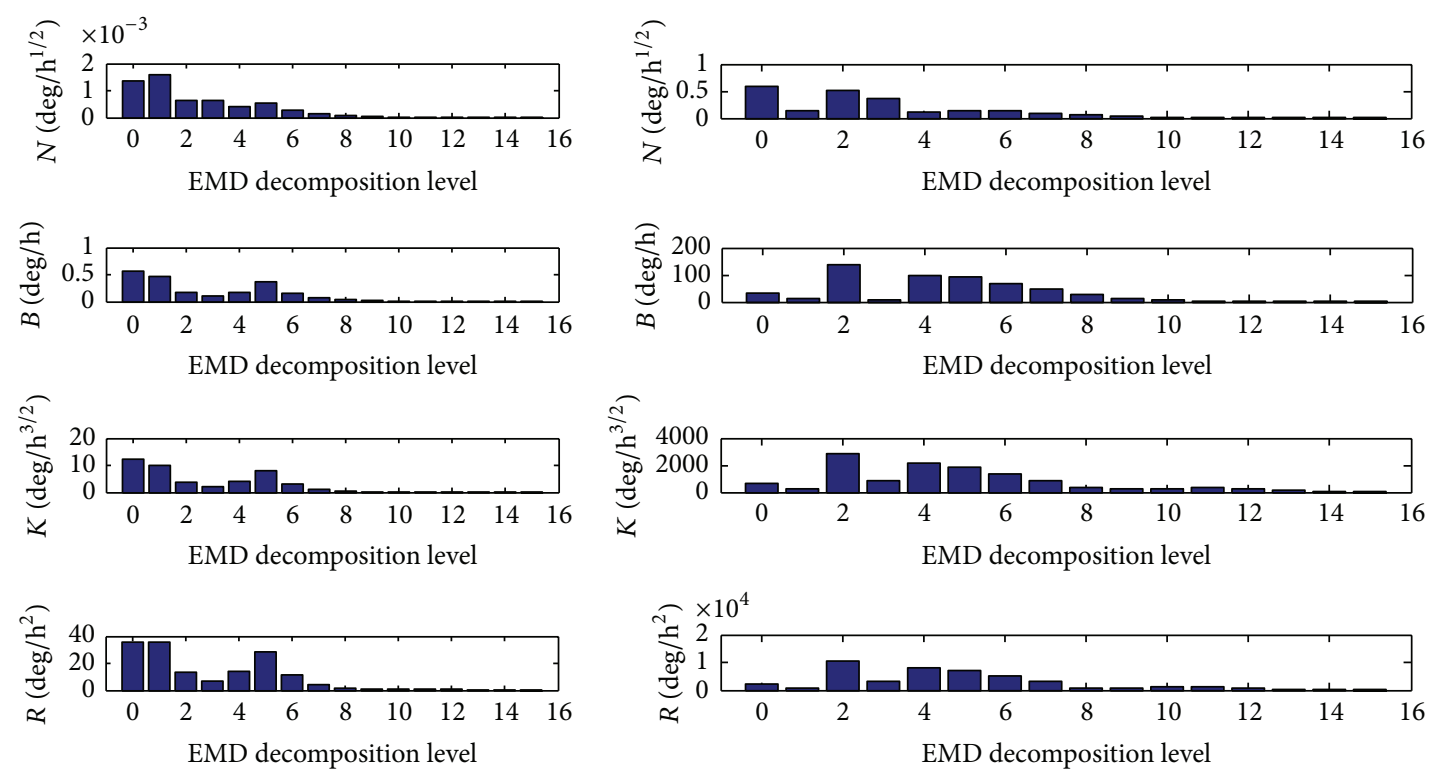

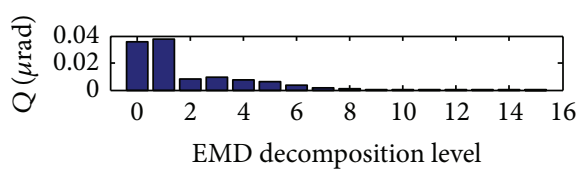

(a)

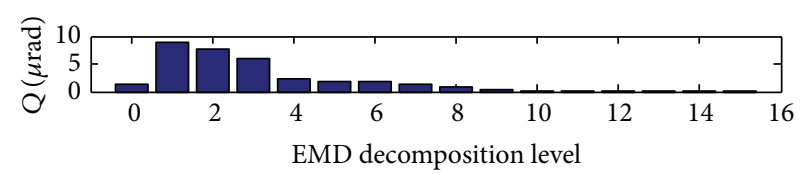

(b)

Figure 5: Relationship between AV coefficients (level 0) and EMDAV coefficients: (a) FOG and (b) MEMS gyro.

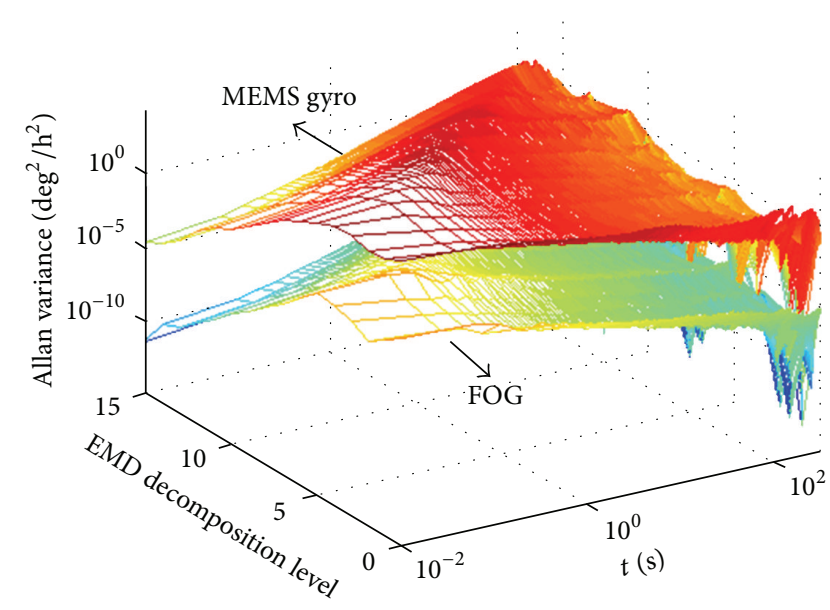

(a)

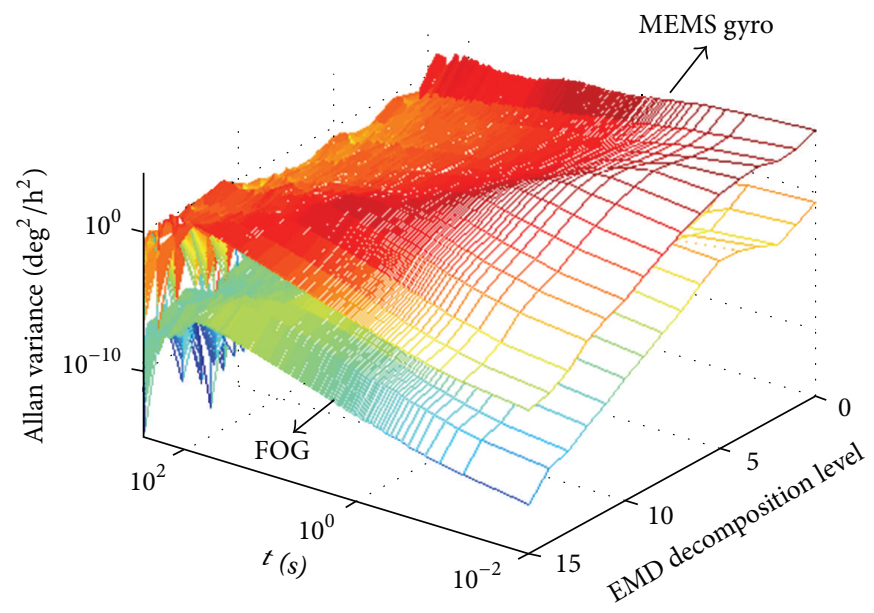

(b)

FIGURE 6: AV and EMDAV 3D logarithmic graph: (a) front view and (b) back view.

\section{Competing Interests}

The authors declare that there is no conflict of interests regarding the publication of this paper.

\section{Acknowledgments}

This research has been mainly funded by the Fundamental Research Funds for the Central Universities (NS2015037) and partly funded by the National Natural Science Foundation of China (Grants nos. 61533008 and 61374115 to Jianye Liu) and Jiangsu Key Laboratory of Internet of Things and Control Technologies.

\section{References}

[1] J. Lai, P. Lv, J. Liu, and B. Jiang, "Noncommutativity error analysis of strapdown inertial navigation system under the vibration in UAVs," International Journal of Advanced Robotic Systems, vol. 9, article 134, 2012. 
[2] R. Peesapati, S. L. Sabat, K. P. Karthik, J. Nayak, and N. Giribabu, "Efficient hybrid Kalman filter for denoising fiber optic gyroscope signal," Optik, vol. 124, no. 20, pp. 4549-4556, 2013.

[3] V. V. Chikovani, "Performance parameters comparison of ring laser, coriolis vibratory and fiber-optic gyros based on Allan variance analysis," in Proceedings of the IEEE 2nd International Conference on Actual Problems of Unmanned Air Vehicles Developments (APUAVD '13), pp. 153-156, IEEE, Kiev, Ukraine, October 2013.

[4] C.-S. Qu, T.-Z. Lu, and Y. Tan, "A modified empirical mode decomposition method with applications to signal de-noising," Acta Automatica Sinica, vol. 36, no. 1, pp. 67-73, 2010.

[5] Y. Gan, L. Sui, G. Xiao, and Y. Duan, "EMD de-noising theory considering static and dynamic conditions and its applications in INS," Lecture Notes in Electrical Engineering, vol. 245, pp. 507515, 2013.

[6] Y. Zhang, S. Wang, and D. Xia, "EMD-based denoising methods in the MEMS gyroscope de-drift," in Proceedings of the 5th IEEE International Conference on Nano/Micro Engineered and Molecular Systems (NEMS '10), pp. 591-594, Xiamen, China, January 2010.

[7] H. Tian, R. Yuan, C. Zhang, J. Jin, N. Song, and S. Lin, "Nonlinear empirical mode predictive drift extraction on fiber optical gyroscope," Optik, vol. 122, no. 5, pp. 415-421, 2011.

[8] S. Jain, S. Nandy, G. Chakraborty, C. S. Kumar, R. Ray, and S. N. Shome, "Error modeling of various sensors for robotics application using allan variance technique," in Proceedings of the IEEE International Conference on Signal Processing, Communications and Computing (ICSPCC '11), pp. 1-4, IEEE, Xi'an, China, September 2011.

[9] N. Zhang and X. Li, "Research on theoretical improvement of dynamic Allan variance and its application," Acta Optica Sinica, vol. 31, no. 11, pp. 70-75, 2011.

[10] J. C. Li, F. Q. Gao, G. L. Wang, W. Gao, W. Zhu, and M. Wang, "Analysis of dynamic Allan variance for fiber optic gyro under vibration and variable temperature conditions," Chinese Journal of Lasers, vol. 40, no. 9, pp. 1-7, 2013.

[11] S. Dang, W. Tian, and F. Qian, "EMD- and LWT-based stochastic noise eliminating method for fiber optic gyro," Measurement, vol. 44, no. 10, pp. 2190-2193, 2011.

[12] N. E. Huang, Z. Shen, S. R. Long et al., "The empirical mode decomposition and the Hilbert spectrum for nonlinear and non-stationary time series analysis," Proceedings of the Royal Society of London A: Mathematical, Physical and Engineering Sciences, vol. 454, no. 1971, pp. 903-995, 1998.

[13] Y. Gan and L. F. Sui, "De-noising method for gyro signal based on EMD," Acta Geodaetica et Cartographica Sinica, vol. 40, no. 6, pp. 745-750, 2011. 


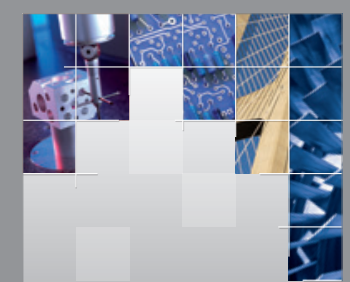

\section{Enfincering}
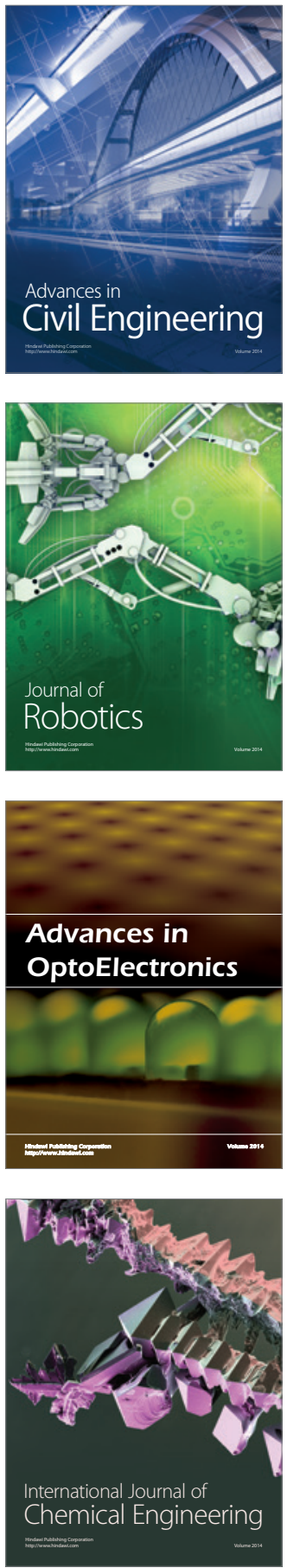

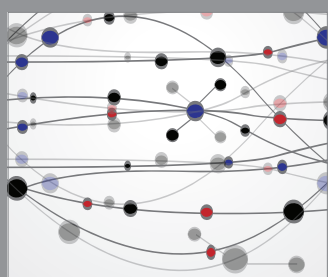

The Scientific World Journal

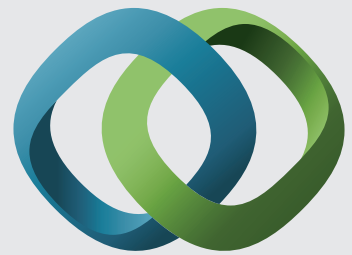

\section{Hindawi}

Submit your manuscripts at

http://www.hindawi.com
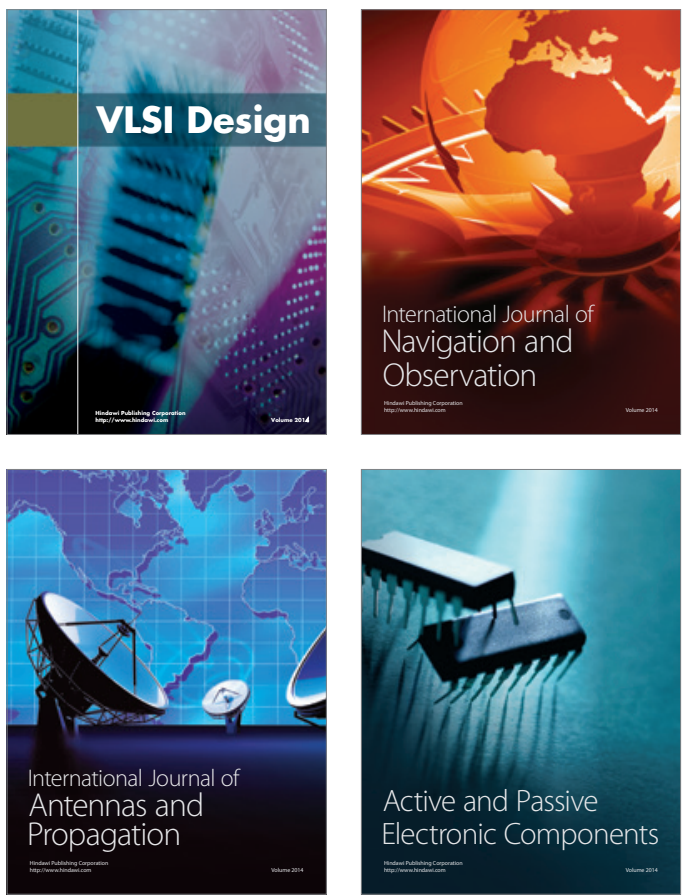
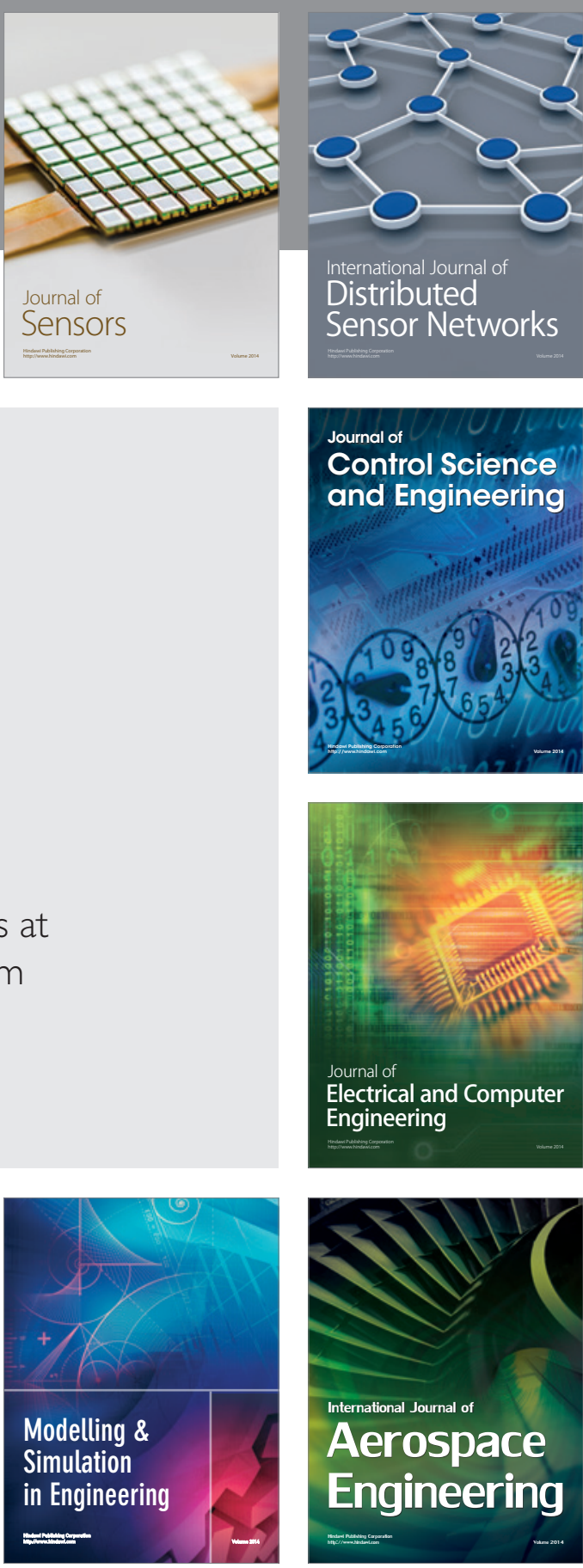

International Journal of

Distributed

Sensor Networks

Journal of

Control Science

and Engineering
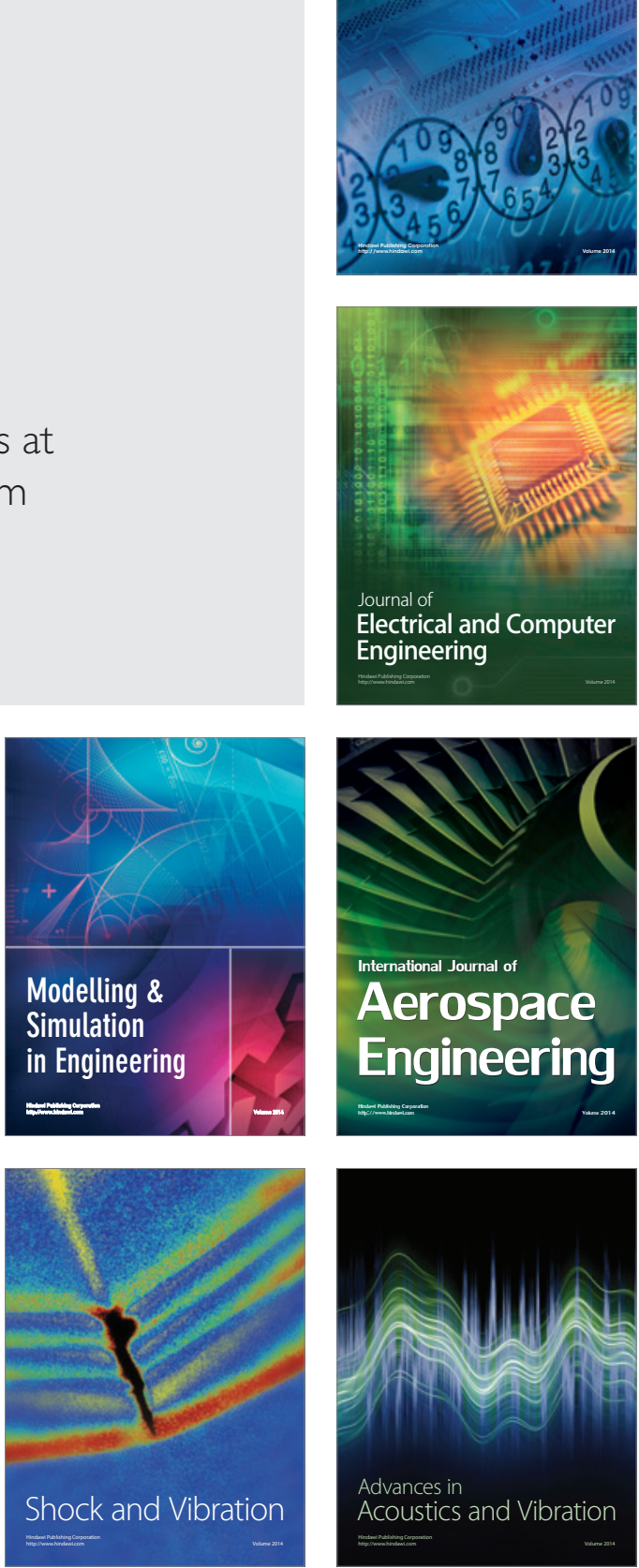